\title{
Effective Remodelling of Human Osteoarthritic Cartilage by sox 9 Gene Transfer and Overexpression upon Delivery of rAAV Vectors in Polymeric Micelles
}

\author{
Ana Rey-Rico, ${ }^{*}, \dagger$ Jagadesh K. Venkatesan, ${ }^{\dagger}$ Gertrud Schmitt, ${ }^{\dagger}$ Susanne Speicher- \\ Mentges, ${ }^{\dagger}$ Henning Madry, ${ }^{\dagger}, \S$ and Magali Cucchiarini ${ }^{\dagger}$ \\ ${ }^{\dagger}$ Center of Experimental Orthopaedics, Saarland University Medical Center, Homburg D-66421, Germany \\ * Centro de Investigacions Cientí ficas Avanzadas (CICA), Universidade da Coruña, Campus de A Coruña, 15071 A \\ Coruña, Spain \\ $\S$ Department of Orthopaedics and Orthopaedic Surgery, Saarland University Medical Center, Homburg D-66421, \\ Germany
}

\begin{abstract}
Recombinant adeno-associated virus (rAAV) vectors are well suited carriers to provide durable treatments for human osteoarthritis (OA). Controlled release of rAAV from polymeric micelles was already shown to increase both the stability and bioactivity of the vectors while overcoming barriers, precluding effective gene transfer. In the present study, we examined the convenience of delivering rAAV vectors via poly(ethylene oxide) (PEO) and poly(propylene oxide) (PPO) polymeric (PEO-PPO-PEO) micelles to transfer and overexpress the transcription factor SOX9 in monolayers of human $\mathrm{OA}$ chondrocytes and in experimentally created human osteochondral defects. Human osteoarthritic (OA) chondrocytes and human osteochondral defect models were produced using human OA cartilage obtained from patients subjected to total knee arthroplasty. Samples were genetically modified by adding a rAAVFLAG-hsox 9 vector in its free form or via polymeric micelles for 10 days relative to control conditions (unmodified cells). The effects of sox 9 overexpression in human OA cartilage samples were monitored by biochemical, histological, and immunohistochemical analyses. Delivery of rAAV-FLAG-hsox9 via polymeric micelles enhanced the levels of sox 9 expression compared with free vector administration, resulting in increased proteoglycan deposition and in a stimulated cell proliferation index in OA chondrocytes. Moreover, higher production of type II collagen and decreased hypertrophic events were noted in osteochondral defect cultures when compared with control conditions. Controlled therapeutic rAAV sox9 gene delivery using PEO-PPO-PEO micelles is a promising, efficient tool to promote the remodelling of human OA cartilage.
\end{abstract}




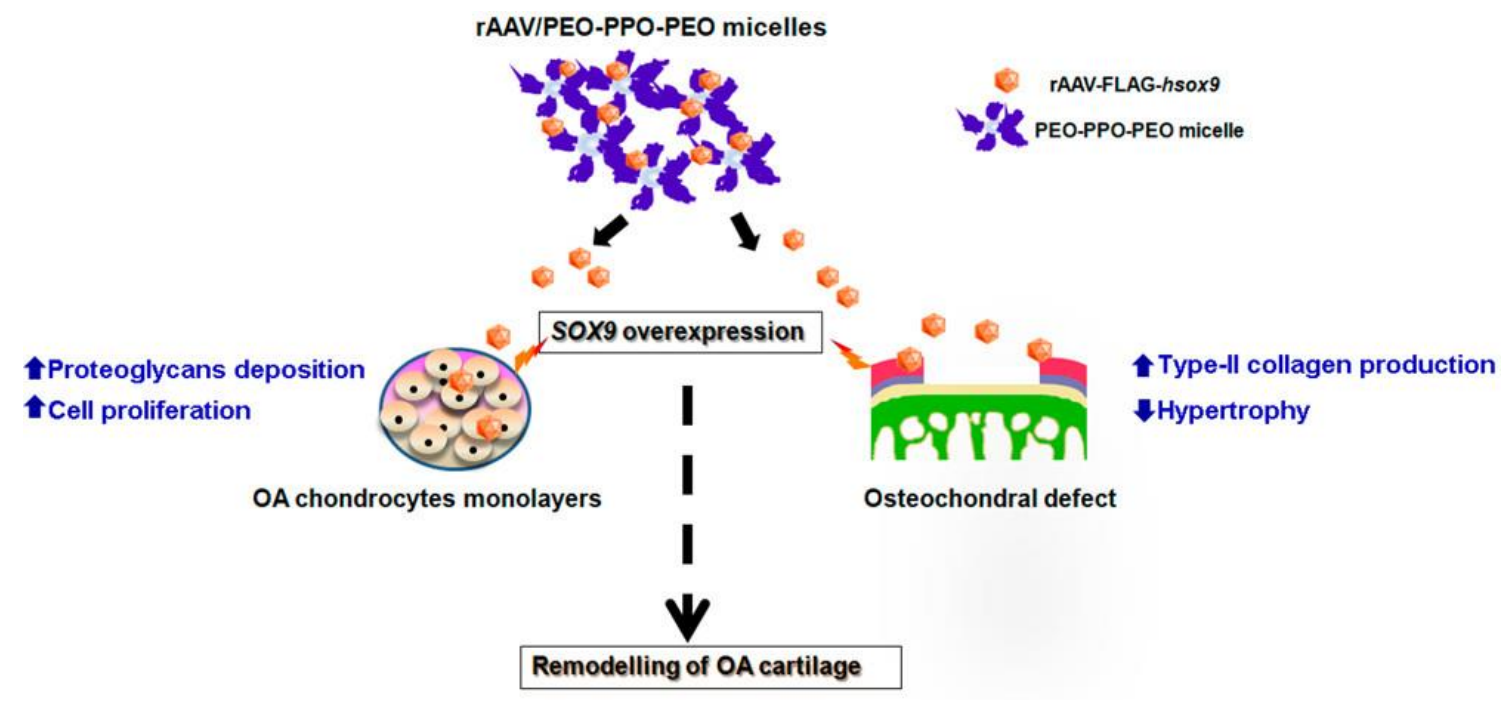

KEYWORDS:

Human osteoarthritic articular cartilage; rAAV vectors; SOX9 overexpression; PEO-PPO-PEO copolymers; polymeric micelles

\section{Introduction}

Osteoarthritis (OA) is a chronic, degenerative joint condition characterized by regressive changes of the articular cartilage (loss of proteoglycans and type II collagen), the remodelling of the subchondral bone, and generation of osteophytes $(1,2)$ due to an imbalanced homeostasis. $(3,4)$

Nonetheless, none of the actual treatments for OA has led to reproduce the native hyaline cartilage integrity in patients. The design of advanced, durable, and effective therapies based on the combination of tissue engineering approaches (5,6) and cellular therapies (7) are therefore under active investigation to impede additional tissue degeneration and to promote the reparative processes at the place of the injuries. Among these lines, gene therapy is a powerful strategy to promote cartilage reparative processes in OA tissue by a direct supply of genes encoding for therapeutic factors in the tissue to be repaired, allowing for the extended synthesis of a candidate agent when compared with the use of short half-life growth factors. $(8,9)$

Therapeutic gene transfer for OA treatment has been already involved to modulate the metabolic functions of OA chondrocytes either to protect OA joints from cartilage disruption by providing sequences with preventive and/or inhibitory activities (IL-1 receptor antagonist, inhibitors of nuclear factor kappa-light-chain-enhancer of activated B cells $(\mathrm{NF}-\kappa \mathrm{B})$, thrombospontin-1, kallistatin, proopiomelanocortin, Dickkopf-1) (10-17) or to promote anabolic processes by using genes encoding for anabolic and/or proliferative factors as the insulin-like growth factor 1 (IGF-1), $(18,19)$ basic fibroblast growth factor (FGF-2), (20) bone morphogenetic proteins (BMPs), (21) and proteoglycan 4. (22)

Other potential approaches to readjust the disturbed cartilage homeostasis include the correction of the gene expression profiles being altered in OA chondrocytes. In this scenario, transcription factors are pivotal regulators of cartilage metabolism as they promote chondrogenesis in both pathologic or physiologic conditions. (23) Among them, SOX9 (SRY-Box 9), a member of the sex-determining region Y-type (SRY) high mobility group box (HMG) family of DNA binding proteins, plays crucial functions in the regulation of skeletal and cartilage biosynthesis (24) and in the formation of permanent 
cartilage. (25) Therefore, the pattern of expression of SOX9 during embryonic development is closely parallel to that of cartilage matrix production, exerting its functions by means of the activation of the gene for type II collagen and other cartilage-specific genes. (26-29) Of note, the expression of SOX9 is notably decreased in OA pathology. $(30,31)$

Genetic modification of the levels of intracellular SOX9 expression constitutes a potential approach to switch the disrupted equilibrium characteristic from OA cartilage toward the production of extracellular matrix (ECM) compounds contributing to the reproduction of an original articular cartilage surface. (23) Recombinant adeno-associated virus (rAAV) vectors are specially adapted vehicles to this end since they can introduce genes into human OA chondrocytes with high efficiencies and for extended periods of time, $(23,32)$ obviating the risks commonly associated with other types of classical vectors. $(8,9)$ Yet, the clinical administration of these vectors in patients might be precluded by the ubiquity of neutralizing antibodies against the AAV viral capsid proteins in the host, (33) particularly by those contained in the synovial fluid from patients with joint pathologies. (34)

Controlled delivery of rAAV vectors via polymeric biomaterials is an emerging, effective tool to tackle those pre-existing hurdles. $(35,36)$ Hereof, we previously reported that encapsulation of rAAV vectors in polymeric micelles built with poly(ethylene oxide) (PEO) and poly(propylene oxide) (PPO) triblock copolymers as linear poloxamers (PF68) or X-shaped poloxamines (T908) enhanced both rAAV stability and bioactivity and afforded a protection of these vectors in conditions of experimental neutralization. (37) Furthermore, overexpression of the transforming growth factor beta (TGF- $\beta$ ) via these systems increased both the deposition of proteoglycans and the cell numbers in human OA chondrocytes in monolayer cultures in vitro and the levels of type II collagen in experimentally created human osteochondral defects. (38)

Based on these results, the goal of the current work was to explore the potential of PF68- and T908-based polymeric micelles to transfer a rAAV-FLAG-hsox 9 vector to both human OA chondrocyte monolayers and human osteochondral defects to overexpress this potent transcription factor as a therapeutic way to remodelling human OA cartilage. The effects of sox 9 overexpression were monitored by biochemical, histological, and immunohistochemical analyses.

\section{Materials and Methods}

\subsection{Materials}

Pluronic F68 and Tetronic 908 were generously supplied by BASF (Ludwigshafen, Germany). Cell proliferation Reagent WST-1 was from Roche Applied Science (Mannheim, Germany). Alcian blue 8GX reagent was obtained from Sigma. The anti-SOX9 (C-20) antibody was acquired from Santa Cruz Biotechnology (Heidelberg, Germany), the antitype II collagen (II-II6B3) antibody from DSHB (Iowa, IA, USA), and the antitype X collagen (COL-10) antibody from Sigma (Munich, Germany). Biotinylated secondary antibodies and the ABC reagent were obtained from Vector Laboratories (Alexis Deutschland $\mathrm{GmbH}$, Grünberg, Germany).

\subsection{Cells and Osteochondral Defect Model}

Human osteoarthritic (OA) cartilage (Mankin score 7-9) was retrieved from patients subjected to total knee arthroplasty $(n=4)$ with previous informed consent signature. (39) The study was authorized by the Ethics Committee of the Saarland Physicians Council (Approval Ha67/12), and all protocols were in agreement with the Helsinki Declaration. Human OA chondrocytes (passage 1-2) were isolated following standard protocols $(38,39)$ and cultured in DMEM, 10\% FBS, $100 \mathrm{U} / \mathrm{mL}$ penicillin $\mathrm{G}, 100 \mu \mathrm{L} / \mathrm{mL}$ streptomycin (growth medium) for $12 \mathrm{~h}$ at $37{ }^{\circ} \mathrm{C}$ under $5 \% \quad \mathrm{CO}_{2}$ previously to the addition of the dispersions of rAAV vectors in free forms or formulated in polymeric micelles. (38) Osteochondral defects were created in human OA cartilage biopsies $(n=2)$ using a $1 \mathrm{~mm}$ biopsy punch in standardized cylindrical $(6 \mathrm{~mm}$ diameter) $(37,38)$ and kept in growth medium previously to the addition of the same samples described above. (38) 


\subsection{Plasmids and rAAV Vectors}

rAAV-FLAG-hsox 9 constructs were derived from pSSV9, an AAV-2 genomic clone, $(40,41)$ and carried a FLAG-tagged human sox 9 cDNA under the control of the cytomegalovirus immediate-early (CMV-IE) promoter. $(23,42-44)$ The vectors were produced as conventional (not self-complementary) vectors by using a helper-free, two-plasmid transfection system in 293 cells with the packaging plasmid pXX2 and the Adenovirus helper plasmid pXX6. (39) The vector preparations were purified by dialysis and titrated by real-time PCR, $(39,45,46)$ showing an average of $10^{10}$ transgene copies $/ \mathrm{mL}(\sim 1 / 500$ functional recombinant viral particles).

\subsection{Preparation of Micellar Solutions Containing rAAV Vectors}

Copolymer samples (PF68 or T908) were dissolved in a given volume of $10 \%$ sucrose aq. solution at 4 ${ }^{\circ} \mathrm{C}$, mixed with rAAV, and incubated in ice-water bath for $30 \mathrm{~min}$ before their use. $(37,46)$ Effective interaction of rAAV vectors with polymeric micelles was confirmed by an increased size of the aggregates as noted by transmission electron microscopy (TEM) and dynamic light scattering (DLS). (37)

\subsection{Gene Transfer Efficiency Using the rAAV-FLAG-hsox9/Polymeric Micelles}

Monolayer cultures of human OA chondrocytes (3000 cells/well or 7500 cells/well for alcian blue staining in 96-well plates) were incubated with the rAAV-FLAG-hsox9/PF68 or rAAV-FLAGhsox9/T908 micelles ( $2 \times 10^{8}$ transgene copies, final copolymer concentration in culture medium $\left.2 \%\right)$ and cultured for 10 days at $37{ }^{\circ} \mathrm{C}$ with three medium changes per week. (38) For osteochondral defect cultures, formulations of rAAV-FLAG-hsox9/PF68 or rAAV-FLAG-hsox9/T908 micelles $(4 \mathrm{x}$ $10^{8}$ transgene copies, final copolymer concentration in culture medium $2 \%$ ) were directly added into the defects, and cultures were maintained for up to 10 days at $37^{\circ} \mathrm{C}$ (38) with three weekly medium changes. Administration of rAAV vectors encoding for a reporter gene (lacZ) was not studied here due to its lack of effects on chondrocytes metabolism. $(37,43)$

\subsection{Detection of Transgene Expression}

SOX9 expression was evaluated by immunocyto/histochemistry by using a specific primary antibody (1:60 dilution) and a biotinylated secondary antibody (1:200 dilution). Immmunodetection was performed using the $\mathrm{ABC}$ method with diaminobenzidine (DAB) as the chromogen. $(42,44)$ Controls of monolayers cultures or sections without primary antibody were studied in parallel. Samples were analyzed with light microscopy (Olympus CKX41 and BX 45, Hamburg, Germany).

\subsection{Assessment of Cell Proliferation and Viability}

Proliferation of OA chondrocytes monolayers was measured by using the Cell Proliferation Reagent WST-1. $(38,45)$ Control conditions included cell monolayers cultured with or without the same dose of free rAAV-FLAG-hsox 9 vector (positive and negatives controls, respectively) and with micellar solution in absence of vector. Optical densities (ODs) at $450 \mathrm{~nm}$ were measured with a GENios spectrophotometer/fluorometer (Tecan, Mainz, Germany), and the percents of cell viabilities were determined as follows:

$$
\text { viability }(\%)=\left[\left(\mathrm{OD}_{\text {sample }}\right) /\left(\mathrm{OD}_{\text {negative control }}\right)\right] \times 100
$$




\subsection{Histological and Immunohistochemical Analyses}

OA chondrocyte monolayers and osteochondral defect cultures were collected after 10 days and fixed in $4 \%$ formalin. $(37,38)$ Alcian blue staining of chondrocytes monolayers was assessed to detect matrix proteoglycans. $(37,47,48)$ In brief, fixed chondocytes monolayers were stained with alcian blue $(1 \%$ in $\mathrm{HCl} 1 \mathrm{~N}$ ) and subsequently washed off with double distilled water. Quantitative estimation of the staining was performed by monitoring the ODs at $595 \mathrm{~nm}$ using a GENios spectrophotometer/fluorometer (Tecan) after overnight solubilization of the samples in $6 \mathrm{M}$ guanidine hydrochloride. Cultures of osteochondral defects were dehydrated, embedded in paraffin, and sectioned at $10 \mu \mathrm{m}$. $(37,38)$ Histological staining of the sections was performed to detect cell structures and densities with hematoxylin eosin (H\&E), matrix proteoglycans (safranin O), and matrix mineralization (alizarin red). (39,45,46) Expression of SOX9 and type II/X collagen was monitored by using specific primary antibodies (dilutions 1:60, 1:10, and 1:200, respectively), biotinylated secondary antibodies (dilution 1:200), and the $\mathrm{ABC}$ method with $\mathrm{DAB}$ as the chromogen, with examination by light microscopy (Olympus CKX41 and BX 45).

\subsection{Histomorphometry}

Cell proliferation (cell number $/ \mathrm{mm}^{2}$ ) on H\&E-stained sections and the staining intensities (pixels) of safranin $\mathrm{O}$ and alizarin red staining and of SOX9 and type II immunostaining were evaluated at 4 randomized places for each test, parameter, and replicate condition. Data were expressed as mean \pm standard deviations (SD). $(37,38)$ The percents of safranin O staining and those of type X collagen immunostaining were calculated by measuring the tissue surface positively stained relative to total surface (pixels) of the place evaluated on histological sections. Analyses were performed with SIS AnalySIS (Olympus), ImageJ, and Adobe Photoshop Adobe Systems Software (Unterschleissheim, Germany). $(37,49,50,38)$

\subsection{Statistical Analysis}

Each condition was carried out in duplicate in two independent experiments. Data are expressed as mean \pm standard deviation (SD). Statistical analysis was accomplished by using SPSS version 23 (IBM, Armonk, NY, USA). One-way analysis of variance (ANOVA) with Tukey's LSD or Games-Howell post hoc tests was involved to evaluate differences between groups. A $p$-value of less than 0.05 was considered statistically significant.

\section{Results}

\subsection{Effective rAAV-Mediated sox 9 Overexpression in Human OA Chondrocytes by Delivery of rAAV Vectors via PEO-PPO-PEO Micelles}

We initially evaluated the ability of PEO-PPO-PEO polymeric micelles to successfully deliver rAAVFLAG-hsox 9 to monolayer cultures of human OA chondrocytes in vitro.

An estimation of the sox 9 expression levels by immunohistochemistry showed an effective rAAV-FLAGhsox9-mediated gene transfer (up to 1.52-fold increase; $p \leq 0.001$ compared with negative control) (Figure 1). Of note, delivery of rAAV vectors via polymeric micelles resulted in the most intense SOX9 immunoreactivity, especially when using PF68-based micelles (up to 1.14-fold increase; $p \leq 0.010$ compared with free vector treatment). 
A

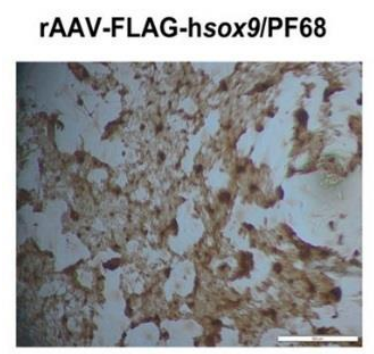

rAAV-FLAG-hsox 9

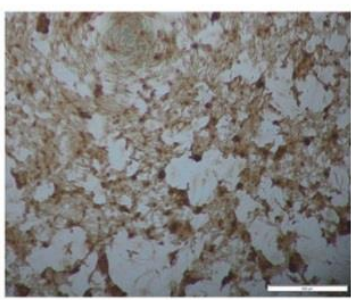

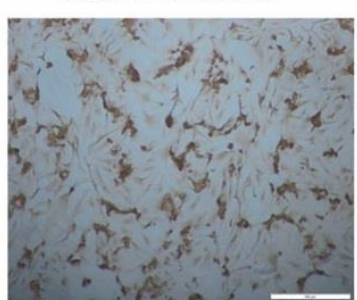

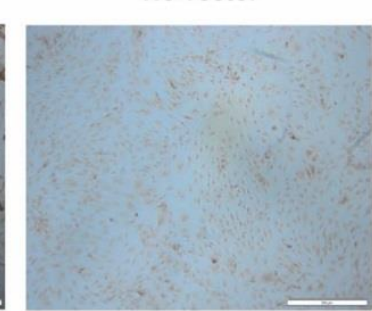

B

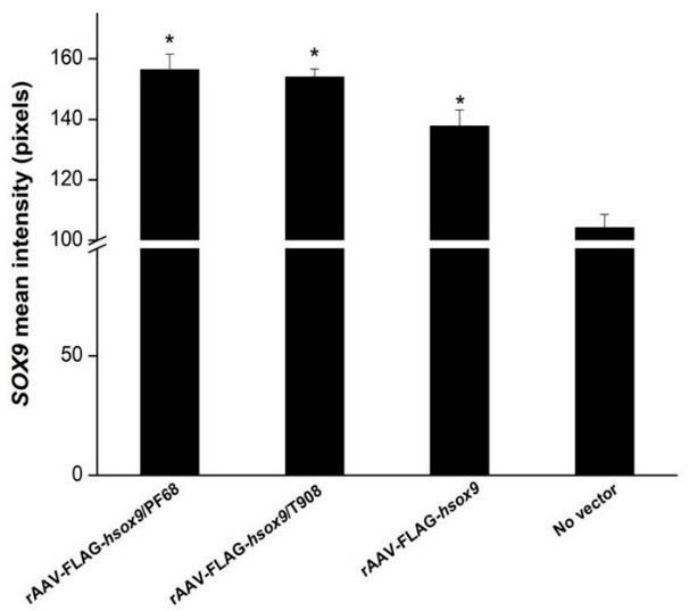

Figure 1. Transgene expression in rAAV-FLAG-hsox9-transduced human OA chondrocytes in the presence of polymeric micelles. Cells in monolayer culture were prepared and incubated with the rAAV/polymeric micelles as described in the Materials and Methods. The cultures were processed after 10 days to monitor (A) SOX9 expression by immunohistochemical detection (magnification $\times 4$, all representative data) with (B) the corresponding histomorphometric analyses as described in the Materials and Methods. Control conditions included the absence of copolymer or vector treatment (negative control) and application of free rAAV vector (positive control). *Statistically significant compared with the negative control.

\subsection{Impact of rAAV-FLAG-hsox9/PEO-PPO-PEO Micelles Delivery on the Biological Activities of Human OA Chondrocytes}

Next, we examined the biological effects of $\operatorname{sox} 9$ overexpression by providing rAAV-FLAG-hsox 9 via PEO-PPO-PEO polymeric micelles in monolayer cultures of human OA chondrocytes.

An analysis of the levels of cell proliferation after 10 days revealed increased proliferative indices following rAAV $\operatorname{sox} 9$ gene transfer (up to 1.4 -fold increase; $p \leq 0.020$ compared with the negative control), particularly by delivery of the vectors via polymeric micelles, albeit statistically significant differences were not reached when compared with free vector administration $(p \geq 0.107)$ (Figure 2A). 

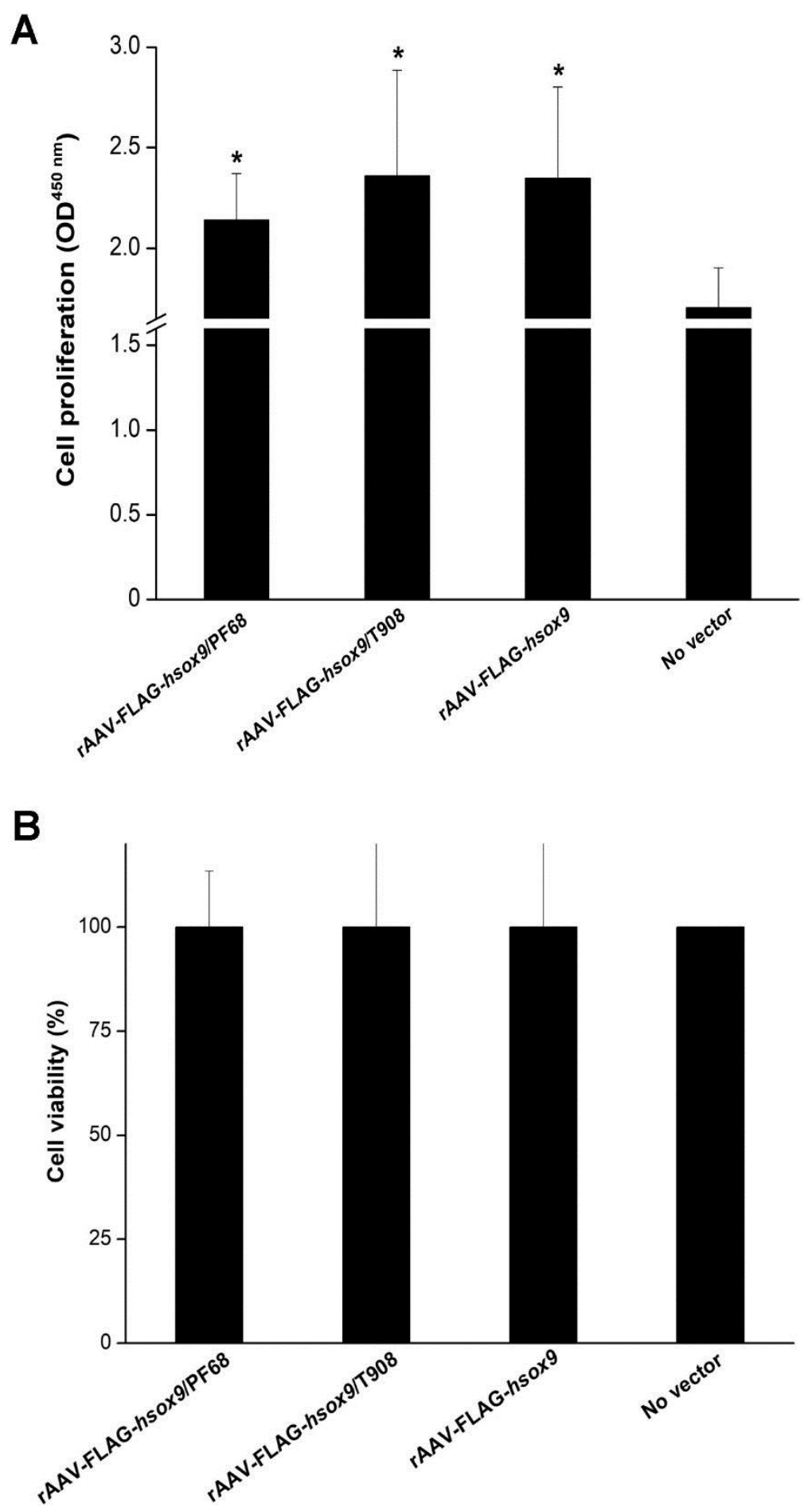

Figure 2. Cell proliferation and viability in rAAV-FLAG-hsox9-transduced human OA chondrocytes in the presence of polymeric micelles. Cells in monolayer cultures were prepared and incubated with the rAAV/polymeric micelles as described in the Materials and Methods. The cultures were processed at the denoted time points to monitor (A) cell proliferation using the WST-1 assay and (B) the \% of cell viability as described in the Materials and Methods. Control conditions included the absence of copolymer or vector treatment (negative control) and application of free rAAV vector (positive control). *Statistically significant compared with the negative control. 
Noteworthy, no cytotoxic effects of any of the gene transfer conditions were noted versus control condition (always $p>0.900$ ) (Figure 2B). A similar trend was noted by administration of copolymer solutions without vector treatment (data not shown).

Lastly, administration of rAAV-FLAG-hsox9 resulted in an increased deposition of proteoglycans compared with untreated cells (up to 1.44 -fold difference, $p \leq 0.038$ versus negative control) (Figure 3A,B). Strikingly, overexpression of sox 9 via rAAV using the polymeric micelles resulted in a higher proteoglycan deposition compared with free rAAV-FLAG-hsox9 administration (up to 1.2-fold increase, $p=0.031$ compared with free vector administration) (Figure 3A,B).

A

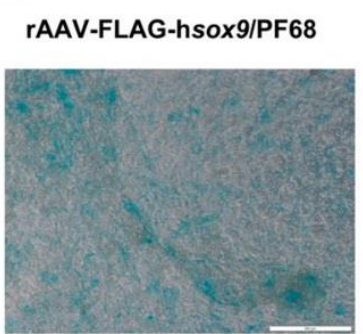

rAAV-FLAG-hsox9/T908

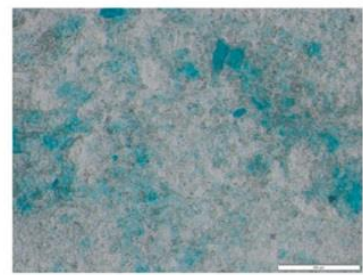

rAAV-FLAG-hsox 9

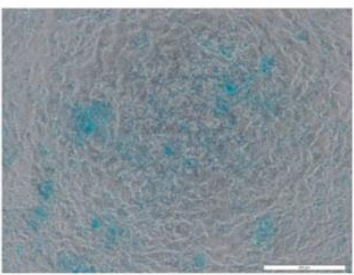

No vector

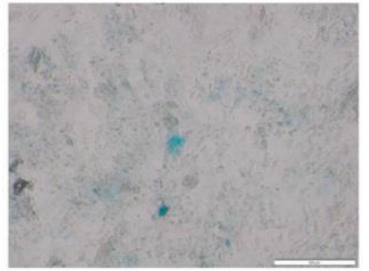

B

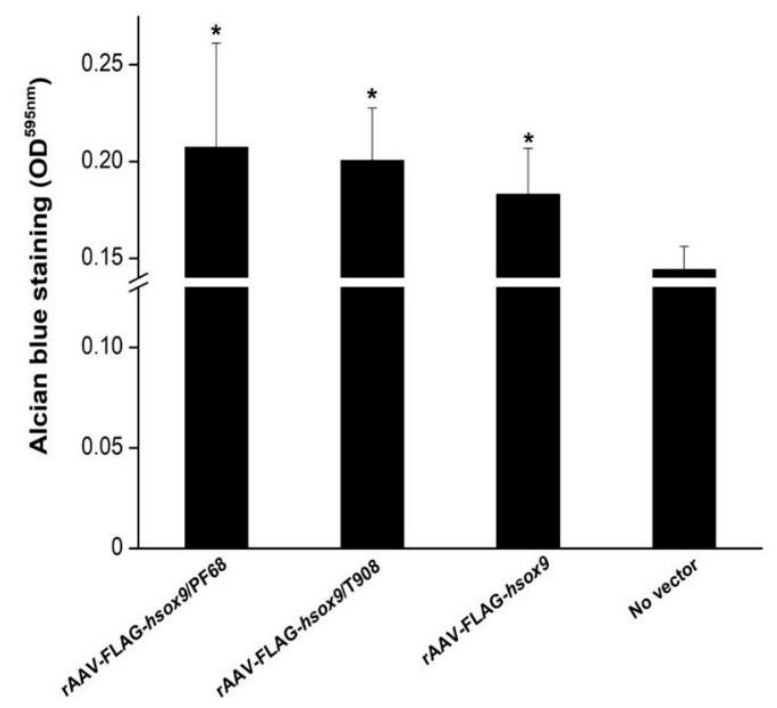

Figure 3. Biosynthetic activities in rAAV-FLAG-hsox9-transduced human OA chondrocytes in the presence of polymeric micelles. Cells in monolayer cultures were prepared and incubated with the rAAV/polymeric micelles as described in the Materials and Methods. The cultures were processed at the denoted time points for (A) alcian blue staining (magnification $\times 10$, all representative data) with (B) spectrophotometric analyses after solubilization in $6 \mathrm{M}$ guanidine hydrochloride as described in the Materials and Methods. Control conditions included the absence of copolymer or vector treatment (negative control) and application of free rAAV vector (positive control). *Statistically significant compared with the negative control. 


\subsection{Effective rAAV-Mediated sox9 Overexpression in Human Osteochondral Defect Cultures by Delivery of rAAV Vectors via PEO-PPO-PEO Micelles}

We next evaluated the ability of PEO-PPO-PEO polymeric micelles to effectively deliver the rAAVFLAG-hsox 9 candidate vector to human osteochondral defect cultures in situ.

In concordance with our findings in monolayer cultures of human OA chondrocytes, efficient $\operatorname{sox} 9$ expression was notified in defect cultures upon rAAV-FLAG-hsox 9 transduction after 10 days (always $p \leq 0.020$ compared with control condition), particularly when providing the vectors via PF68-based micelles (up to 1.1-fold difference versus control condition, $p \leq 0.001$ ), although statistical significant differences were not reached relative to free vector administration $(p \leq 0.430)$ (Figure 4).

\section{A}

PF68/rAAV-FLAG-hsox9 T908/rAAV-FLAG-hsox9 rAAV-FLAG-hsox 9
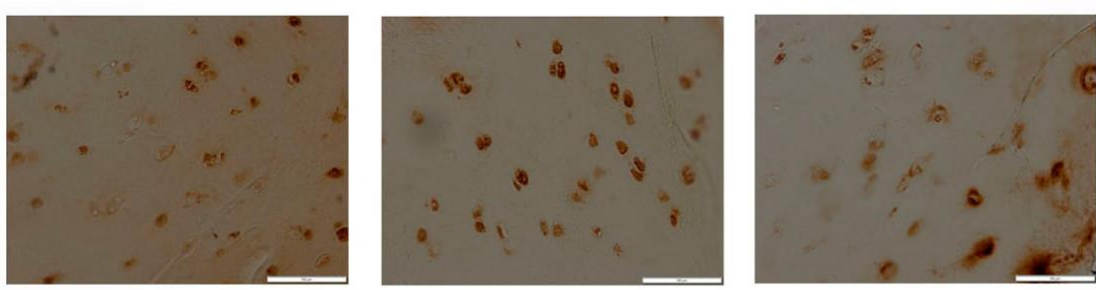

B

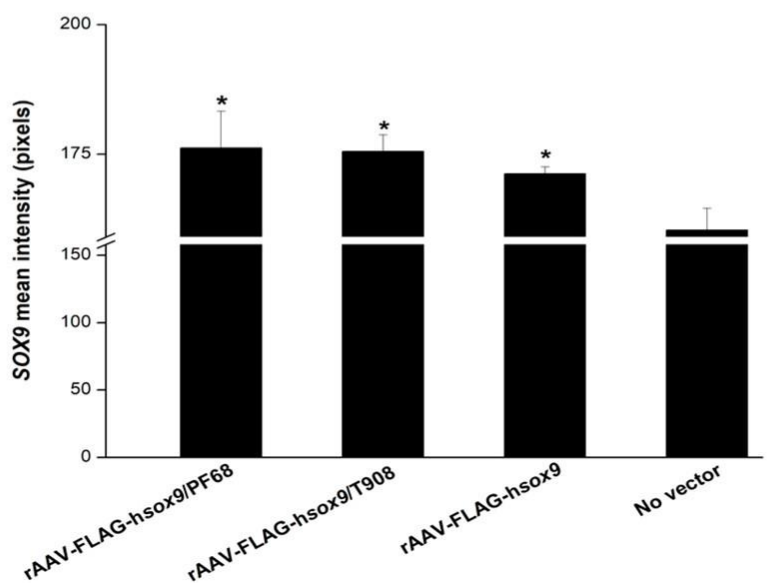

No vector

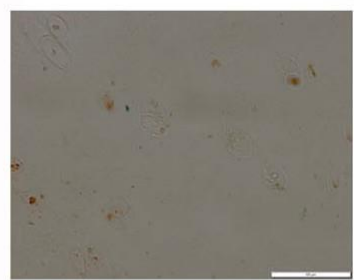

Figure 4. Transgene expression in rAAV-FLAG-hsox9-transduced human osteochondral defect cultures in the presence of polymeric micelles. Osteochondral defects were prepared and incubated with the rAAV/polymeric micelles as described in the Materials and Methods. The cultures were processed after 10 days to monitor (A) SOX9 expression by immunohistochemical detection (magnification $\times 20$, all representative data) with (B) the corresponding histomorphometric analyses as described in the Materials and Methods. Control conditions included the absence of copolymer or vector treatment (negative control) and application of free rAAV vector (positive control). *Statistically significant compared with the negative control. 


\subsection{Impact of rAAV-FLAG-hsox9/PEO-PPO-PEO Micelles Delivery on the Remodelling Activities of Human Osteochondral Defect Cultures}

We last investigated the effects of sox 9 overexpression in cultures of human osteochondral defects by applying rAAV-FLAG-hsox9 in PEO-PPO-PEO polymeric micelles.

An evaluation of the cell densities in regions contiguous to the defects on H\&E-stained sections always revealed significantly higher cell contents following sox 9 overexpression (always $p \leq 0.088$ compared with vector condition), especially when rAAV-FLAG-hsox9 was provided via PF68-based micelles (up to 2.6-fold difference versus control condition, $p \leq 0.012$ ) (Figure $5 \mathrm{~A}, \mathrm{~B}$ ).

A

rAAV-FLAG-hsox9/PF68 rAAV-FLAG-hsox9/T908
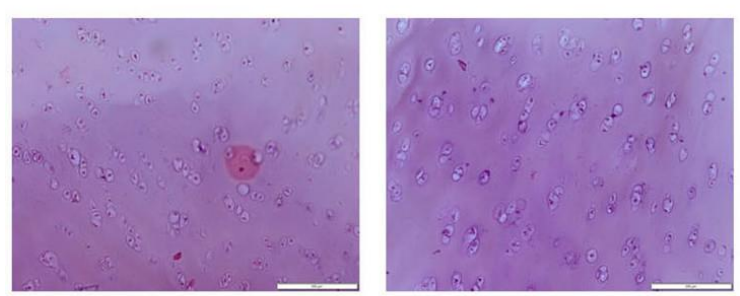

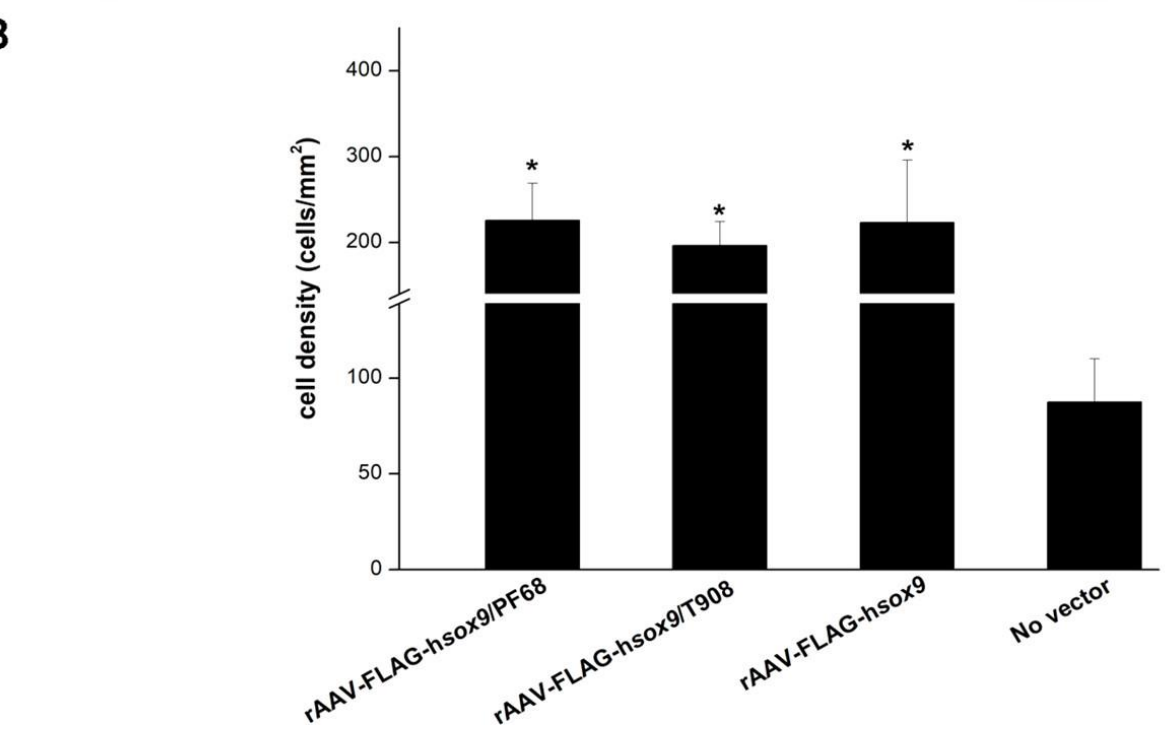

rAAV-FLAG-hsox 9

No vector
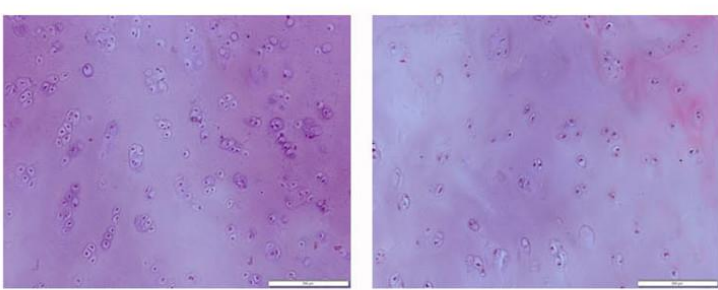

B

Figure 5. Cell viability in rAAV-FLAG-hsox9-transduced human osteochondral defect cultures in the presence of polymeric micelles. Osteochondral defects were prepared and incubated with the rAAV/polymeric micelles as described in the Materials and Methods. The cultures were processed after 10 days for $(\mathrm{A}) \mathrm{H} \& \mathrm{E}$ staining (magnification $\times 10$, all representative data) with (B) the corresponding histomorphometric analyses as described in the Materials and Methods. Control conditions included the absence of copolymer or vector treatment (negative control) and application of free rAAV vector (positive control). *Statistically significant compared with the negative control. 
Likewise, rAAV-mediated sox 9 gene transfer significantly increased the production of a proteoglycanrich ECM as noted by the higher percents of safranin $\mathrm{O}$-stained surface in treated defects relative to untreated control samples (up to 1.25-fold difference, $\mathrm{p}=0.0001$ ) (Figure 6A,B). Interestingly, the defects treated with free rAAV-FLAG-hsox9 displayed the highest percents of stained surface (up to 1.16-fold difference, $\mathrm{p} \leq 0.035$ compared with the administration of rAAV in polymeric micelles). An estimation of the intensities safranin $O$ staining in the defects revealed a similar tendency, with the highest intensities detected in defects treated with free rAAV-FLAG-hsox9 (up to 1.15-fold difference, $p$ $=0.005$ compared with control condition) (Figure 6A,C). Finally, no differences in the safranin $\mathrm{O}$ intensities were noted, independently of the route of rAAV-FLAG-hsox9 administration (free vector or administration in polymeric micelles) $(\mathrm{p} \geq 0.069)$. 
A

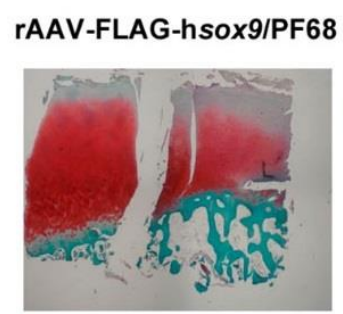

B

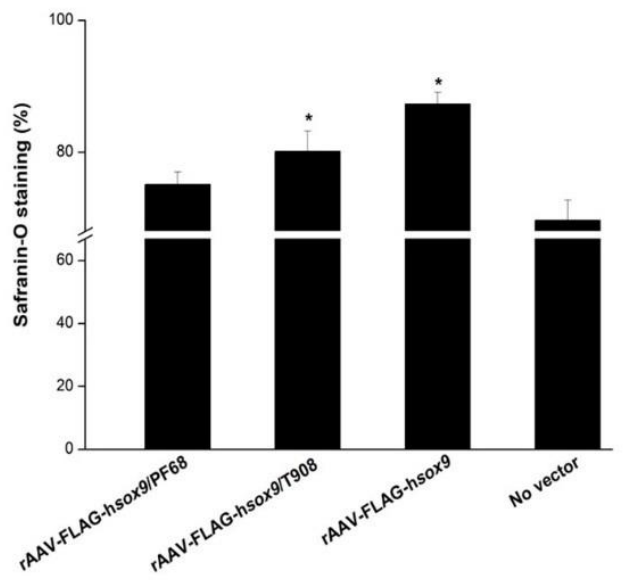

rAAV-FLAG-hsox 9

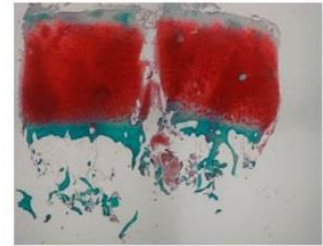

C

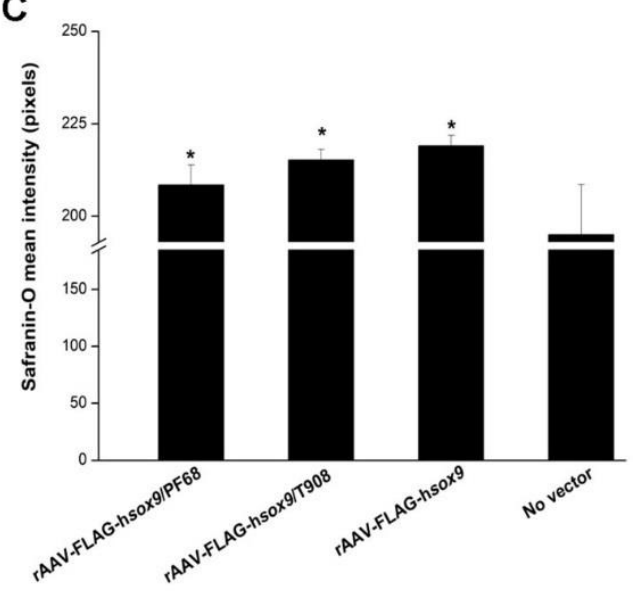

No vector

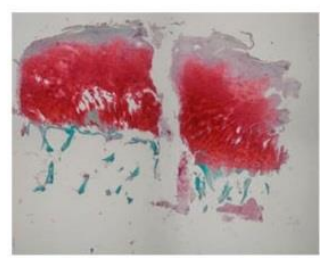

D

rAAV-FLAG-hsox9/PF68

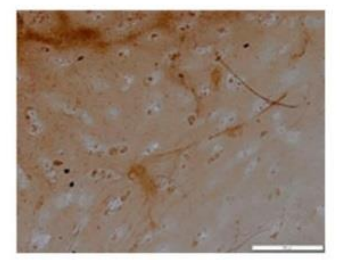

E
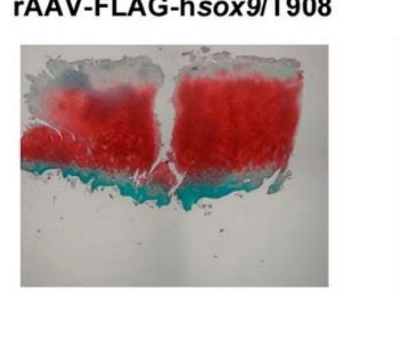
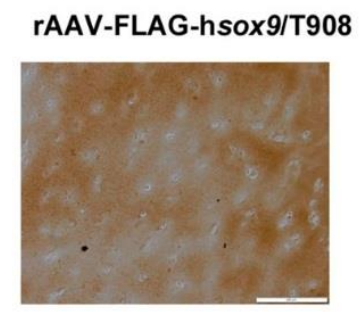

rAAV-FLAG-hsox 9

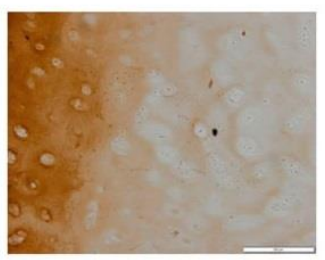
. 
Of further note, administration of rAAV-FLAG-hsox 9 also prompted significantly higher amounts of type II collagen deposition compared with control conditions (always $p<0.009$ ), particularly by delivery via T908 micelles (up to 1.35-fold difference, $p=0.005$ ) (Figure 6C,D).

Remarkably, an estimation of the percents of type X collagen expression in regions close to the defects showed a significant decrease of hypertrophy upon rAAV-FLAG-hsox9-mediated gene transfer (up to 26.3-fold difference relative to control condition, $p \leq 0.009$ ), while no differences were detected in groups treated with rAAV-FLAG-hsox9 $(p \geq 0.930)$ (Figures 7A,B). Similarly, an estimation of alizarin red staining intensity in the neighboring regions to the defects revealed a significant decrease of matrix mineralization upon rAAV-FLAG-hsox9-mediated transduction (up to 1.2-fold difference relative to control condition, $p \leq 0.034$ ) (Figure 6C,D). However, no differences were observed between the groups treated where rAAV-FLAG-hsox 9 was provided, regardless of the delivery approach involved (polymeric micelles, free vector administration) $(p \geq 0.410)$. 
A

rAAV-FLAG-hsox9/PF68

rAAV-FLAG-hsox9/T908

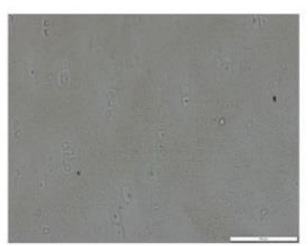

B

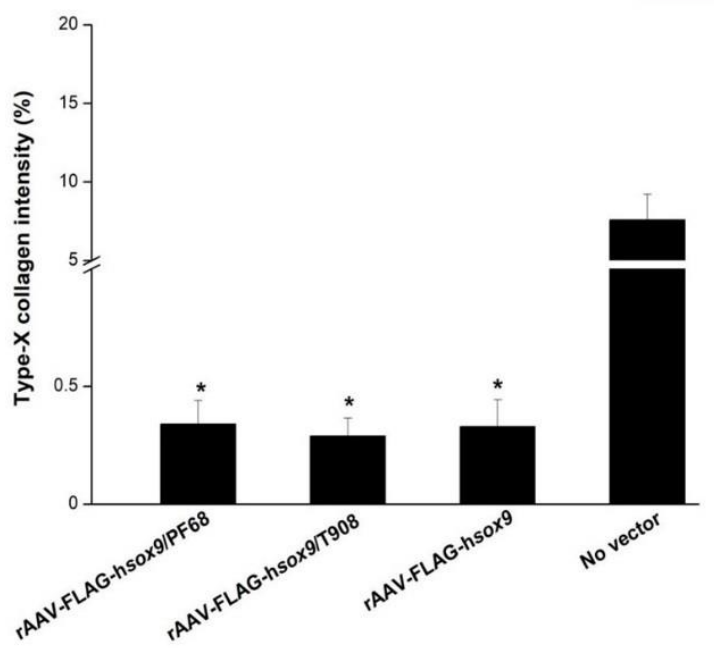

C

rAAV-FLAG-hsox9/PF68

rAAV-FLAG-hsox9/T908

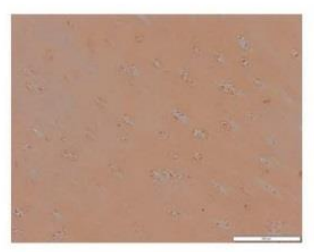

rAAV-FLAG-hsox 9
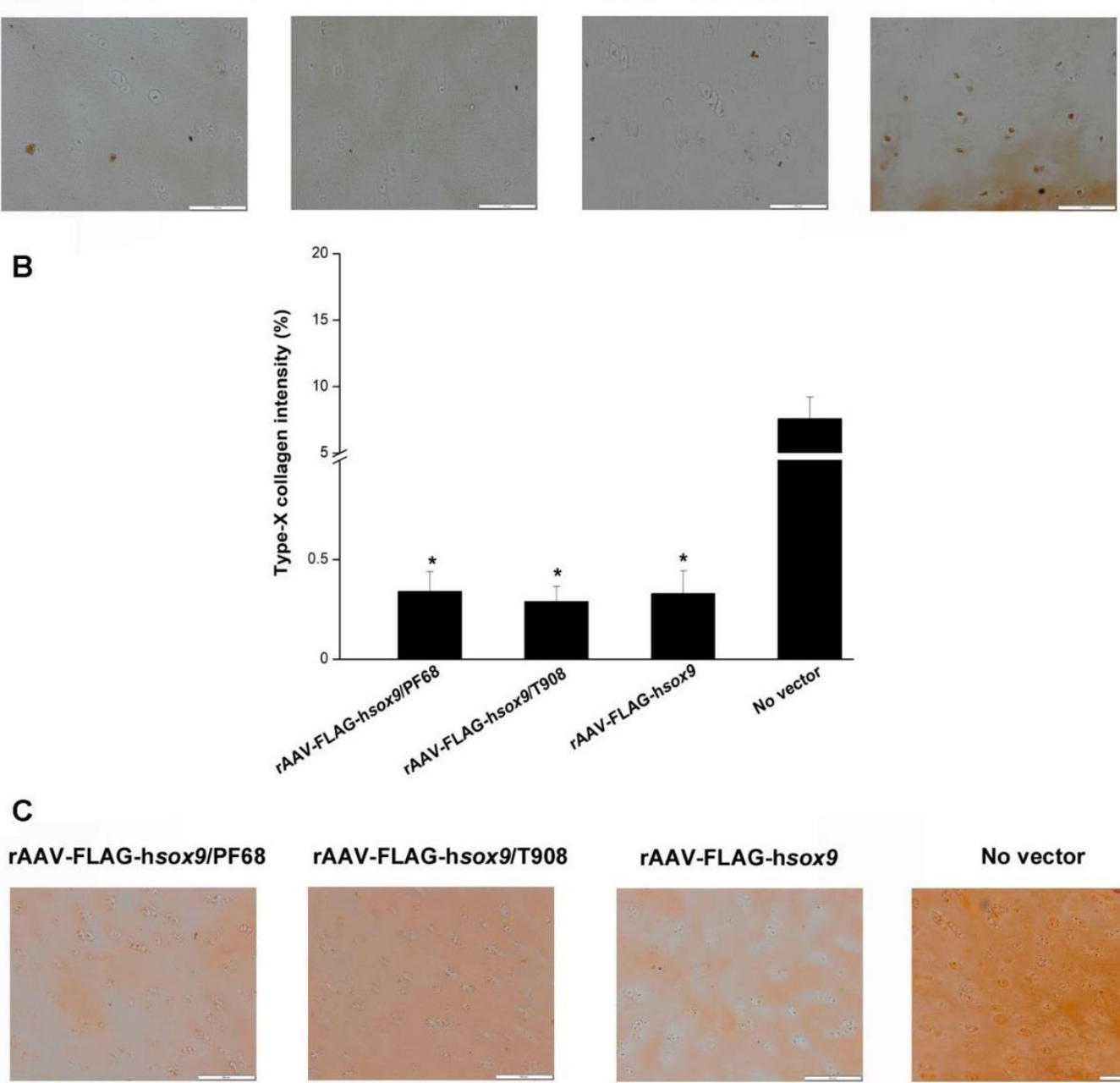

D

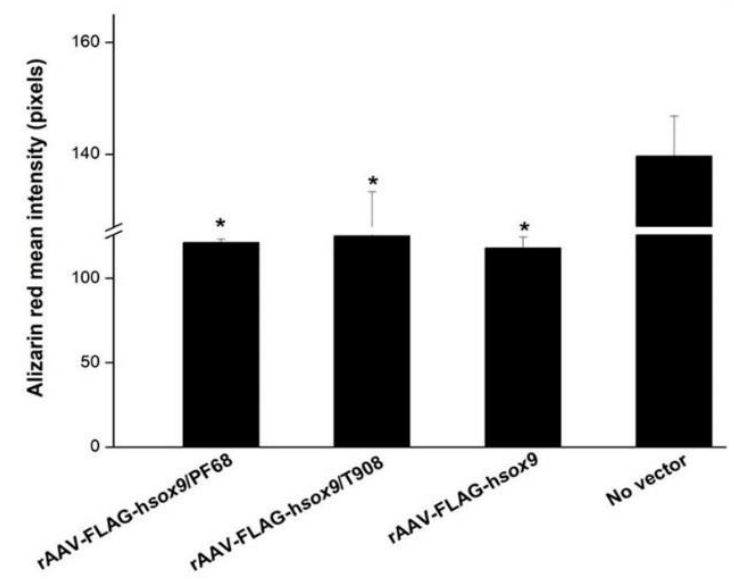

Figure 7. Hypertrophic and terminal differentiation processes in rAAV-FLAG-hsox9-transduced human osteochondral defect cultures in the presence of polymeric micelles. Osteochondral defects were prepared and incubated with the rAAV/polymeric micelles as described in the Materials and Methods. The cultures were processed after 10 days for (A) immunohistochemical detection of type $\mathrm{X}$ collagen (magnification $\times 20$, all representative data) with (B) the corresponding histomorphometric analyses and for $(C)$ alizarin red staining (magnification $\times 10$, all representative data) with (D) the corresponding histomorphometric analyses as described in the Materials and Methods. Control conditions included the absence of copolymer or vector treatment (negative control) and application of free rAAV vector (positive control). *Statistically significant compared with the negative control. 


\section{Discussion}

Gene transfer mediated by rAAV vectors constitutes a powerful approach for the treatment of progressive human disorders like OA due to the ability of these vectors to stably transduce injured articular cartilage in situ $(19,32,39)$ when compared with alternative, less efficient gene delivery systems (nonviral, adenoviral, and retroviral vectors). $(8,9)$ Still, the clinical application of rAAV may still be precluded by the ubiquity of neutralizing antibodies in individuals as those existing in the synovial fluid from patients with joint disorders. (34) Remarkably, delivery of rAAV via PEO-PPO-PEO polymeric micelles was already reported as a potent approach to overcome such limitations. $(37,46)$ Specifically, providing rAAV vectors encoding for a reporter gene (lacZ) via PF68 and T908 micelles has shown to enhance the stability and bioactivity of the vectors, prompting higher levels of lacZ expression in human OA chondrocytes in either normal or gene transfer inhibition situation. (37) Furthermore, overexpression of TGF- $\beta$ via delivery of rAAV vectors via similar systems increased both the deposition of proteoglycans and the cells numbers in human OA chondrocyte monolayer cultures, besides the deposition of type II collagen in human osteochondral defects. (38) In the present work and based on our previous findings, we evaluated the advantages of delivering $\mathrm{rAAV}$ vectors encoding for the potent transcription factor SOX9 in human OA chondrocytes via these PEO-PPO-PEO-based polymeric micelles.

Our results first show that supply of rAAV-FLAG-hsox9 via polymeric micelles to human OA chondrocytes resulted in increased levels of $\operatorname{sox} 9$ transgene expression over time when compared with free vector administration. Moreover, in good agreement with previous studies, rAAVmediated sox 9 gene transfer of human OA chondrocytes led to increased cell proliferation index, (23) especially upon delivery of the vectors via polymeric micelles. (38) Of note, no detrimental effects of any of the rAAV delivery approaches (polymeric micelles, free vector administration) were noted relative to the control condition. The data further indicate that overexpression of $\operatorname{sox} 9$ in human OA chondrocytes resulted in a higher deposition of a proteoglycan-rich ECM compared with untreated cells. Noteworthy, the controlled delivery of rAAV-FLAG-hsox9 in polymeric micelles was more effective to increase the production of proteoglycans in human OA chondrocytes compared with the administration of the free form of the vector.

In order to establish the translatability of the system to target human OA chondrocytes in a more natural and clinically relevant environment, we next delivered rAAV-FLAG-hsox9 formulated in PEO-PPOPEO micelles to a human osteochondral defect model. $(37,38)$ In good concordance with our observations in monolayer culture, efficient SOX9 expression was noted in defect cultures upon rAAV-FLAGhsox9 application, notably when providing the vector via PF68-based micelles. Of note, an estimation of the cell densities in regions close to the defects showed significantly higher cell numbers upon sox 9 overexpression. This is concordant with the previous observation that overexpression of sox9 increases the cellularity in human normal and OA cartilage explant cultures. $(23,43)$ Remarkably, the highest levels of SOX9 expression in OA chondrocyes detected by providing rAAV-FLAG-hsox9 via PF68-based polymeric micelles resulted in the highest cell densities. Next, we report that transfer of rAAV-FLAG-hsox9 either in its free form or via polymeric micelles enhanced the proteoglycan contents in human osteochondral defect cultures. Administration of the rAAV sox 9 construct also prompted significantly higher type II collagen deposition levels, another key compound from the ECM, (51) particularly by delivery via T908 micelles. These results highlight the ability of SOX9 to remodel human $\mathrm{OA}$ cartilage by means of the activation of the biological activities from chondrocytes. (29) Finally, and in good agreement with our observations in rabbit osteochondral defects in vivo, (52) a favorable decrease of hypertrophic events was observed upon rAAV-FLAG-hsox9mediated transduction in human osteochondral defect cultures.

In conclusion, the current work demonstrate the benefits of using PF68- and T908-based micelles as efficient rAAV delivery systems to overexpress the therapeutic transcription factor SOX9 both in monolayers of human OA chondrocytes and in experimental human osteochondral defects for enhanced biological and reparative responses. 


\title{
5. Conclusions
}

The present findings highlight the potential of providing therapeutic rAAV vectors via controlled delivery systems to enhance both spatial and temporal presentation of the gene product into the targets, opening a way to counteract the progression of OA. Specifically here we demonstrated that encapsulation of rAAV carrying a sequence coding for the potent transcription factor SOX9 in systems based on poly(ethylene oxide) (PEO) and poly(propylene oxide) (PPO) triblock copolymers as linear poloxamers (PF68) or Xshaped poloxamines (T908) allowed to promote higher levels of sox9 transgene expression both in chondrocyte monolayers and in experimental osteochondral defects. Notably, bioactivity of rAAVFLAG-hsox 9 provided via polymeric micelles was evidenced as seen by increases in proteoglycan deposition and in cell densities both in vitro and in experimental osteochondral defects. Such promising findings form a basis to develop adapted, vector controlled release treatments against human OA in a close future. However, additional studies in clinically relevant animal models of cartilage defects are still needed to assess the potential of polymeric micelles to stimulate the natural reparative processes in places of injured tissue.

\section{Author Information}

\section{Corresponding Author}

Ana Rey-Rico - Center of Experimental Orthopaedics, Saarland University Medical Center, Homburg D66421, Germany; Centro de Investigacións Científicas Avanzadas (CICA), Universidade da Coruña, Campus de A Coruña, 15071 A Coruña, Spain; http://orcid.org/0000-0003-1682-

498X; Email: ana.rey.rico@gmail.com

\author{
Authors \\ Jagadesh K. Venkatesan - Center of Experimental Orthopaedics, Saarland University Medical Center, \\ Homburg D-66421, Germany \\ Gertrud Schmitt - Center of Experimental Orthopaedics, Saarland University Medical Center, Homburg \\ D-66421, Germany \\ Susanne Speicher-Mentges - Center of Experimental Orthopaedics, Saarland University Medical Center, \\ Homburg D-66421, Germany \\ Henning Madry - Center of Experimental Orthopaedics, Saarland University Medical Center, Homburg \\ D-66421, Germany; Department of Orthopaedics and Orthopaedic Surgery, Saarland University \\ Medical Center, Homburg D-66421, Germany \\ Magali Cucchiarini-Center of Experimental Orthopaedics, Saarland University Medical Center, \\ Homburg D-66421, Germany
}

\section{Notes}

The authors declare no competing financial interest.

\section{Acknowledgments}

This work was supported by a grant from Deutsche Forschungsgemeinschaft (DFG RE 328/2-1 to A.R.R., H.M., M.C.). The authors are grateful to R. J. Samulski (The Gene Therapy Center, University of North Carolina, Chapel Hill, NC), X. Xiao (The Gene Therapy Center, University of Pittsburgh, Pittsburgh, PA), and E. F. Terwilliger (Division of Experimental Medicine, Harvard Institutes of Medicine and Beth Israel Deaconess Medical Center, Boston, MA) for supplying the genomic AAV-2 plasmid clones, the pXX2 and pXX6 plasmids, and the 293 cell line. The authors also thank G. Scherer (Institute for Human Genetics and Anthropology, Albert-Ludwig University, Freiburg, Germany) for providing the human $\operatorname{sox} 9 \mathrm{cDNA}$. 


\section{References}

(1) Loeser, R. F.; Goldring, S. R.; Scanzello, C. R.; Goldring, M. B. Osteoarthritis: a disease of the joint as an organ. Arthritis Rheum. 2012, 64 (6), 1697-707, DOI: 10.1002/art.34453

(2) Poole, A. R. Osteoarthritis as a whole joint disease. HSS J. 2012, 8 (1), 4- 6, DOI: 10.1007/s11420-011-9248-6

(3) Kapoor, M.; Martel-Pelletier, J.; Lajeunesse, D.; Pelletier, J. P.; Fahmi, H. Role of proinflammatory cytokines in the pathophysiology of osteoarthritis. Nat. Rev. Rheumatol. 2011, 7 (1), 33- 42, DOI: 10.1038/nrrheum.2010.196

(4) Goldring, M. B.; Otero, M. Inflammation in osteoarthritis. Curr. Opin. Rheumatol. 2011, 23 (5), 471-8, DOI: 10.1097/BOR.0b013e328349c2b1

(5) Szychlinska, M. A.; Castrogiovanni, P.; Nsir, H.; Di Rosa, M.; Guglielmino, C.; Parenti, R.; Calabrese, G.; Pricoco, E.; Salvatorelli, L.; Magro, G.; Imbesi, R.; Mobasheri, A.; Musumeci, G. Engineered cartilage regeneration from adipose tissue derived-mesenchymal stem cells: A morphomolecular study on osteoblast, chondrocyte and apoptosis evaluation. Exp. Cell Res. 2017, 357 (2), 222-235, DOI: 10.1016/j.yexcr.2017.05.018

(6) Gardner, O. F. W.; Musumeci, G.; Neumann, A. J.; Eglin, D.; Archer, C. W.; Alini, M.; Stoddart, M. J. Asymmetrical seeding of MSCs into fibrin-poly(ester-urethane) scaffolds and its effect on mechanically induced chondrogenesis. J. Tissue Eng. Regener. Med. 2017, 11 (10), 2912-2921, DOI: 10.1002/term.2194

(7) Szychlinska, M. A.; Stoddart, M. J.; D’Amora, U.; Ambrosio, L.; Alini, M.; Musumeci, G. Mesenchymal Stem Cell-Based Cartilage Regeneration Approach and Cell Senescence: Can. We Manipulate Cell Aging and Function?. Tissue Eng., Part B 2017, 23 (6), 529- 539, DOI: 10.1089/ten.teb.2017.0083

(8) Madry, H.; Cucchiarini, M. Advances and challenges in gene-based approaches for osteoarthritis. $J$. Gene Med. 2013, 15 (10), 343- 55, DOI: 10.1002/jgm.2741

(9) Evans, C. H.; Huard, J. Gene therapy approaches to regenerating the musculoskeletal system. Nat. Rev. Rheumatol. 2015, 11 (4), 234-42, DOI: 10.1038/nrrheum.2015.28

(10) Pelletier, J. P.; Caron, J. P.; Evans, C.; Robbins, P. D.; Georgescu, H. I.; Jovanovic, D.; Fernandes, J. C.; Martel-Pelletier, J. In vivo suppression of early experimental osteoarthritis by interleukin-1 receptor antagonist using gene therapy. Arthritis Rheum. 1997, 40 (6), 1012- 9, DOI: 10.1002/art.1780400604

(11) Frisbie, D. D.; Ghivizzani, S. C.; Robbins, P. D.; Evans, C. H.; Mcllwraith, C. W. Treatment of experimental equine osteoarthritis by in vivo delivery of the equine interleukin-1 receptor antagonist gene. Gene Ther. 2002, 9 (1), 12-20, DOI: 10.1038/sj.gt.3301608

(12) Chen, L. X.; Lin, L.; Wang, H. J.; Wei, X. L.; Fu, X.; Zhang, J. Y.; Yu, C. L. Suppression of early experimental osteoarthritis by in vivo delivery of the adenoviral vector-mediated NF-kappaBp65-specific siRNA. Osteoarthritis Cartilage 2008, 16 (2), 174- 84, DOI: 10.1016/j.joca.2007.06.006

(13) Hsieh, J. L.; Shen, P. C.; Shiau, A. L.; Jou, I. M.; Lee, C. H.; Wang, C. R.; Teo, M. L.; Wu, C. L. Intraarticular gene transfer of thrombospondin-1 suppresses the disease progression of experimental osteoarthritis. J. Orthop. Res. 2010, 28 (10), 1300-6, DOI: 10.1002/jor.21134

(14) Hsieh, J. L.; Shen, P. C.; Shiau, A. L.; Jou, I. M.; Lee, C. H.; Teo, M. L.; Wang, C. R.; Chao, J.; Chao, L.; Wu, C. L. Adenovirus-mediated kallistatin gene transfer ameliorates disease progression in a rat model of osteoarthritis induced by anterior cruciate ligament transection. Hum. Gene Ther. 2009, 20 (2), 14758, DOI: $10.1089 /$ hum.2008.096

(15) Shen, P. C.; Shiau, A. L.; Jou, I. M.; Lee, C. H.; Tai, M. H.; Juan, H. Y.; Lin, P. R.; Liu, G. S.; Wu, C. L.; Hsieh, J. L. Inhibition of cartilage damage by pro-opiomelanocortin prohormone overexpression in a rat model of osteoarthritis. Exp. Biol. Med. (London, U. K.) 2011, 236 (3), 334- 40, DOI: 10.1258/ebm.2010.010319

(16) Oh, H.; Chun, C. H.; Chun, J. S. Dkk-1 expression in chondrocytes inhibits experimental osteoarthritic cartilage destruction in mice. Arthritis Rheum. 2012, 64 (8), 2568-78, DOI: 10.1002/art.34481

(17) Santangelo, K. S.; Nuovo, G. J.; Bertone, A. L. In vivo reduction or blockade of interleukin-1beta in primary osteoarthritis influences expression of mediators implicated in pathogenesis. Osteoarthritis Cartilage 2012, 20 (12), 1610- 8, DOI: 10.1016/j.joca.2012.08.011

(18) Nixon, A. J.; Goodrich, L. R.; Scimeca, M. S.; Witte, T. H.; Schnabel, L. V.; Watts, A. E.; Robbins, P. D. Gene therapy in musculoskeletal repair. Ann. N. Y. Acad. Sci. 2007, 1117, 310- 27, DOI: 10.1196/annals.1402.065

(19) Weimer, A.; Madry, H.; Venkatesan, J. K.; Schmitt, G.; Frisch, J.; Wezel, A.; Jung, J.; Kohn, D.; Terwilliger, E. F.; Trippel, S. B.; Cucchiarini, M. Benefits of recombinant adeno-associated virus (rAAV)-mediated insulinlike growth factor I (IGF-I) overexpression for the long-term reconstruction of human osteoarthritic cartilage by modulation of the IGF-I axis. Mol. Med. 2012, 18, 346-58

(20) Cucchiarini, M.; Terwilliger, E. F.; Kohn, D.; Madry, H. Remodelling of human osteoarthritic cartilage by FGF-2, alone or combined with Sox9 via rAAV gene transfer. J. Cell Mol. Med. 2009, 13 (8B), 2476- 88, DOI: 10.1111/j.1582-4934.2008.00474.x 
(21) Matsumoto, T.; Cooper, G. M.; Gharaibeh, B.; Meszaros, L. B.; Li, G.; Usas, A.; Fu, F. H.; Huard, J. Cartilage repair in a rat model of osteoarthritis through intraarticular transplantation of muscle-derived stem cells expressing bone morphogenetic protein 4 and soluble Flt-1. Arthritis Rheum. 2009, 60 (5), 1390 405, DOI: $10.1002 /$ art.24443

(22) Ruan, M. Z.; Erez, A.; Guse, K.; Dawson, B.; Bertin, T.; Chen, Y.; Jiang, M. M.; Yustein, J.; Gannon, F.; Lee, B. H. Proteoglycan 4 expression protects against the development of osteoarthritis. Sci. Transl. Med. 2013, 5 (176), 176ra34, DOI: 10.1126/scitranslmed.3005409

(23) Cucchiarini, M.; Thurn, T.; Weimer, A.; Kohn, D.; Terwilliger, E. F.; Madry, H. Restoration of the extracellular matrix in human osteoarthritic articular cartilage by overexpression of the transcription factor SOX9. Arthritis Rheum. 2007, 56 (1), 158-67, DOI: 10.1002/art.22299

(24) Bi, W.; Deng, J. M.; Zhang, Z.; Behringer, R. R.; de Crombrugghe, B. Sox9 is required for cartilage formation. Nat. Genet. 1999, 22 (1), 85-9, DOI: 10.1038/8792

(25) Ikeda, T.; Kamekura, S.; Mabuchi, A.; Kou, I.; Seki, S.; Takato, T.; Nakamura, K.; Kawaguchi, H.; Ikegawa, S.; Chung, U. I. The combination of SOX5, SOX6, and SOX9 (the SOX trio) provides signals sufficient for induction of permanent cartilage. Arthritis Rheum. 2004, 50 (11), 3561-73, DOI: 10.1002/art.20611

(26) Bell, D. M.; Leung, K. K.; Wheatley, S. C.; Ng, L. J.; Zhou, S.; Ling, K. W.; Sham, M. H.; Koopman, P.; Tam, P. P.; Cheah, K. S. SOX9 directly regulates the type-II collagen gene. Nat. Genet. 1997, 16 (2), 174- 8, DOI: 10.1038/ng0697-174

(27) Ng, L. J.; Wheatley, S.; Muscat, G. E.; Conway-Campbell, J.; Bowles, J.; Wright, E.; Bell, D. M.; Tam, P. P.; Cheah, K. S.; Koopman, P. SOX9 binds DNA, activates transcription, and coexpresses with type II collagen during chondrogenesis in the mouse. Dev. Biol. 1997, 183 (1), 108-21, DOI: 10.1006/dbio.1996.8487

(28) Lefebvre, V.; Huang, W.; Harley, V. R.; Goodfellow, P. N.; de Crombrugghe, B. SOX9 is a potent activator of the chondrocyte-specific enhancer of the pro alpha1(II) collagen gene. Mol. Cell. Biol. 1997, 17 (4), 2336- 46, DOI: 10.1128/MCB.17.4.2336

(29) Xie, W. F.; Zhang, X.; Sakano, S.; Lefebvre, V.; Sandell, L. J. Trans-activation of the mouse cartilage-derived retinoic acid-sensitive protein gene by Sox9. J. Bone Miner. Res. 1999, 14 (5), $757-$ 63, DOI: 10.1359/jbmr.1999.14.5.757

(30) Salminen, H.; Vuorio, E.; Saamanen, A. M. Expression of Sox9 and type IIA procollagen during attempted repair of articular cartilage damage in a transgenic mouse model of osteoarthritis. Arthritis Rheum. 2001, 44 (4), 947- 55, DOI: 10.1002/1529-0131(200104)44:4<947::AID-ANR152>3.0.CO;2-4

(31) Aigner, T.; Gebhard, P. M.; Schmid, E.; Bau, B.; Harley, V.; Poschl, E. SOX9 expression does not correlate with type II collagen expression in adult articular chondrocytes. Matrix Biol. 2003, 22 (4), 36372, DOI: $10.1016 / \mathrm{S} 0945-053 \mathrm{X}(03) 00049-0$

(32) Madry, H.; Cucchiarini, M.; Terwilliger, E. F.; Trippel, S. B. Recombinant adeno-associated virus vectors efficiently and persistently transduce chondrocytes in normal and osteoarthritic human articular cartilage. Hum. Gene Ther. 2003, 14 (4), 393-402, DOI: 10.1089/104303403321208998

(33) Calcedo, R.; Wilson, J. M. Humoral Immune Response to AAV. Front. Immunol. 2013, 4, 341, DOI: 10.3389/fimmu.2013.00341

(34) Cottard, V.; Valvason, C.; Falgarone, G.; Lutomski, D.; Boissier, M. C.; Bessis, N. Immune response against gene therapy vectors: influence of synovial fluid on adeno-associated virus mediated gene transfer to chondrocytes. J. Clin. Immunol. 2004, 24 (2), 162- 9, DOI: 10.1023/B:JOCI.0000019781.64421.5c

(35) Rey-Rico, A.; Cucchiarini, M. Controlled release strategies for rAAV-mediated gene delivery. Acta Biomater. 2016, 29, 1- 10, DOI: 10.1016/j.actbio.2015.10.015

(36) Rey-Rico, A.; Cucchiarini, M. Recent tissue engineering-based advances for effective rAAVmediated gene transfer in the musculoskeletal system. Bioengineered 2016, 7, 175, DOI: 10.1080/21655979.2016.1187347

(37) Rey-Rico, A.; Frisch, J.; Venkatesan, J. K.; Schmitt, G.; Rial-Hermida, I.; Taboada, P.; Concheiro, A.; Madry, H.; Alvarez-Lorenzo, C.; Cucchiarini, M. PEO-PPO-PEO Carriers for rAAV-Mediated Transduction of Human Articular Chondrocytes in Vitro and in a Human Osteochondral Defect Model. ACS Appl. Mater. Interfaces 2016, 8 (32), 20600- 13, DOI: 10.1021/acsami.6b06509

(38) Rey-Rico, A.; Venkatesan, J. K.; Schmitt, G.; Concheiro, A.; Madry, H.; Alvarez-Lorenzo, C.; Cucchiarini, M. rAAV-mediated overexpression of TGF-beta via vector delivery in polymeric micelles stimulates the biological and reparative activities of human articular chondrocytes in vitro and in a human osteochondral defect model. Int. J. Nanomed. 2017, 12, 6985-6996, DOI: 10.2147/IJN.S144579

(39) Venkatesan, J. K.; Rey-Rico, A.; Schmitt, G.; Wezel, A.; Madry, H.; Cucchiarini, M. rAAVmediated overexpression of TGF-beta stably restructures human osteoarthritic articular cartilage in situ. $J$. Transl. Med. 2013, 11, 211, DOI: 10.1186/1479-5876-11-211

(40) Samulski, R. J.; Chang, L. S.; Shenk, T. Helper-free stocks of recombinant adeno-associated viruses: normal integration does not require viral gene expression. J. Virol 1989, 63 (9), 3822- 8 
(41) Samulski, R. J.; Chang, L. S.; Shenk, T. A recombinant plasmid from which an infectious adenoassociated virus genome can be excised in vitro and its use to study viral replication. $J$.

Virol 1987, 61 (10), 3096- 101

(42) Venkatesan, J. K.; Ekici, M.; Madry, H.; Schmitt, G.; Kohn, D.; Cucchiarini, M. SOX9 gene transfer via safe, stable, replication-defective recombinant adeno-associated virus vectors as a novel, powerful tool to enhance the chondrogenic potential of human mesenchymal stem cells. Stem Cell Res.

Ther. 2012, 3 (3), 22, DOI: $10.1186 /$ scrt113

(43) Tao, K.; Rey-Rico, A.; Frisch, J.; Venkatesan, J. K.; Schmitt, G.; Madry, H.; Lin, J.; Cucchiarini, M. rAAV-mediated combined gene transfer and overexpression of TGF-beta and SOX9 remodels human osteoarthritic articular cartilage. J. Orthop. Res. 2016, 34, 2181, DOI: 10.1002/jor.23228

(44) Venkatesan, J. K.; Frisch, J.; Rey-Rico, A.; Schmitt, G.; Madry, H.; Cucchiarini, M. Impact of mechanical stimulation on the chondrogenic processes in human bone marrow aspirates modified to overexpress sox9 via rAAV vectors. J. Exp Orthop 2017, 4 (1), 22, DOI: 10.1186/s40634-017-0097-1

(45) Frisch, J.; Venkatesan, J. K.; Rey-Rico, A.; Schmitt, G.; Madry, H.; Cucchiarini, M. Determination of the chondrogenic differentiation processes in human bone marrow-derived mesenchymal stem cells genetically modified to overexpress transforming growth factor-beta via recombinant adeno-associated viral vectors. Hum. Gene Ther. 2014, 25 (12), 1050-60, DOI: 10.1089/hum.2014.091

(46) Rey-Rico, A.; Venkatesan, J. K.; Frisch, J.; Rial-Hermida, I.; Schmitt, G.; Concheiro, A.; Madry, H.; Alvarez-Lorenzo, C.; Cucchiarini, M. PEO-PPO-PEO micelles as effective rAAV-mediated gene delivery systems to target human mesenchymal stem cells without altering their differentiation potency. Acta Biomater. 2015, 27, 42, DOI: 10.1016/j.actbio.2015.08.046

(47) Stanton, L. A.; Sabari, S.; Sampaio, A. V.; Underhill, T. M.; Beier, F. p38 MAP kinase signalling is required for hypertrophic chondrocyte differentiation. Biochem. J. 2004, 378, 53-62, DOI: $10.1042 / \mathrm{bj} 20030874$

(48) Woods, A.; Wang, G.; Beier, F. RhoA/ROCK signaling regulates Sox9 expression and actin organization during chondrogenesis. J. Biol. Chem. 2005, 280 (12), 11626- 34, DOI: 10.1074/jbc.M409158200

(49) Schneider, C. A.; Rasband, W. S.; Eliceiri, K. W. NIH Image to ImageJ: 25 years of image analysis. Nat. Methods 2012, 9 (7), 671- 5, DOI: 10.1038/nmeth.2089

(50) Katagiri, H.; Mendes, L. F.; Luyten, F. P. Definition of a Critical Size Osteochondral Knee Defect and its Negative Effect on the Surrounding Articular Cartilage in the Rat. Osteoarthritis Cartilage 2017, 25 (9), 1531-1540, DOI: 10.1016/j.joca.2017.05.006

(51) Poole, A. R.; Kojima, T.; Yasuda, T.; Mwale, F.; Kobayashi, M.; Laverty, S. Composition and structure of articular cartilage: a template for tissue repair. Clin. Orthop. Relat. Res. 2001, 391, S2633, DOI: 10.1097/00003086-200110001-00004

(52) Cucchiarini, M.; Orth, P.; Madry, H. Direct rAAV SOX9 administration for durable articular cartilage repair with delayed terminal differentiation and hypertrophy in vivo. J. Mol. Med. (Heidelberg, Ger.) 2013, 91 (5), 625-36, DOI: 10.1007/s00109-012-0978-9 